\title{
Medial Subtalar Dislocation in a Basketball Player: Case Report and Literature Review
}

\author{
J alal $Y^{1 *}$, Zaimi $\mathrm{S}^{2}$, Ouzaa $\mathrm{MR}^{1}$, Zine $\mathrm{A}^{1}$ and J aafar \\ $\mathbf{A}^{\mathbf{1}}$ \\ ${ }^{1}$ Department of Orthopedic Surgery and Traumatology, \\ Military Hospital Mohammed V, Morocco \\ ${ }^{2}$ Department of Radiology, Military Hospital Mohamed V, \\ Morocco \\ *Correspondling author: Youssef J alal, Department of \\ Orthopedic Surgery and Traumatology, Military Hospital \\ Mohamed V, Faculty of Medicine and Pharmacy, Rabat, \\ Morocco
}

Received: October 18, 2017; Accepted: November 21, 2017; Published: November 28, 2017

\begin{abstract}
We report a case of purely ligamentous medial subtalar dislocations with closed reduction and conservative treatment, with a cast immobilization. Medial subtalar dislocations following sports injuries are uncommon and rarely reported in the literature, and have an excellent prognosis with early reduction. In the examined case, the dislocation was resulted following landing from a rebound, with the right foot being forced mainly into hyperplantar flexion and eversion. Closed reduction was followed by cast immobilization for 4 weeks. Two years later, the patient had a full range of motion without any pain, while there were no signs of residual instability or early post-traumatic osteoarthritis.
\end{abstract}

We discuss in details the mechanism of such an injury and we highlight the importance of prompt closed reduction and early mobilization to ensure a satisfactory long-term outcome.

Keywords: Medial dislocation; Purely ligamentous; Reduction

\section{Introduction}

Subtalar Dislocation (SD) accounts for only an estimated 1-2\% of all joint dislocations, making it one of the rarest forms of orthopedics injuries [1]. This entity is defined as a simultaneous dislocation of the distal articulations of the talus at both the talocalcaneal and talonavicular joints. It occurs in active young men and its result of a high-energy trauma such as falls from a height, or motorcycle accidents [2]. However, it is not commonly seen as a sports injury because it requires transfer of a high energy. Depending on the position of the foot at the time of injury, Broca (1852) distinguished three types of subtalar dislocation: the medial dislocation, the lateral, and the posterior dislocation. In 1856, Malgaigne and Burger described the fourth type: anterior dislocation [3]. Of all types of subtalar dislocation, medial form is the most frequent, accounting for $79.5 \%$ of all cases, and rest are mostly lateral SD, with only occasional reports of anterior and posterior SD [4]. Those injuries are frequently associated with talar fracture, malleolus fracture, or fracture of the fifth metatarsal bone. However, less than one third of such dislocations are isolated.

We present a case of isolated medial subtalar dislocation in basketball player to discuss the mechanism of such an injury and to highlight the importance of prompt closed reduction and early mobilization to improve the functional outcome after an isolated SD.

\section{Case Presentation}

A 22-year-old basketball player was brought to our accident and emergency department with pain and spectacular deformity of the right foot following landing from a rebound, with the right foot being forced mainly into hyperplantar flexion and eversion (Figure 1). Local examination noticed slightly swollen right ankle, with minimal ecchymosis, and the ankle was found fixed in medial plantar flexion. Pulse of the posterior tibial and dorsalispedis artery were present but weak. General somatic exploration was normal. He subsequently received a radiological assessment that objectified isolated medial subtalar dislocation (Figures 2 \& 3). A closed reduction under sedation was attempted in the emergency room. Thus, firm manual foot traction with counter-traction on the leg followed by eversion of the foot, with direct digital pressure over the head of talus was accomplished. An audible clunk confirmed successful reduction. Once reduced (Figures $4 \& 5$ ), no residual subluxation was noticed and the patient was discharged and immobilized in a short-leg posterior plaster splint for 4 weeks. At his fourth week post-reduction, partial weight bearing was initiated, and active exercise program was started.

Two years later, the patient was satisfied on his function and stability and felt they are adequate to perform job related activities and activities of daily living.

\section{Discussion}

Subtalar dislocations, also known as peritalar dislocations, subastragalar dislocations, or luxatiopedissubtalo, are rare presentations in the emergency department [5]. Judey and Dufaurets

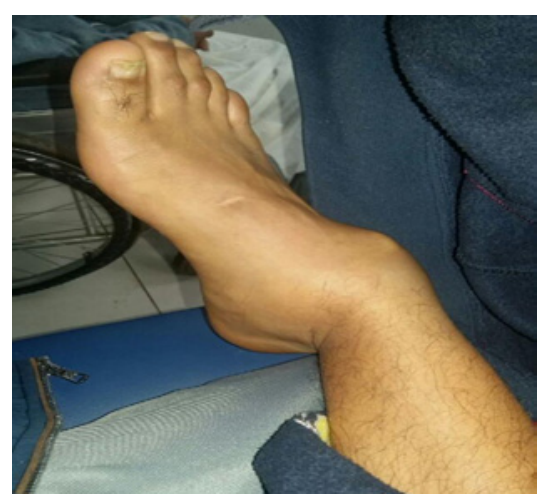

Figure 1: Clinical appearance of a medialsubtalar dislocation with the foot medial to the lower leg and a laterally prominent talar head.
Austin J Orthopade \& Rheumatol - Volume 4 Issue 3 - 2017 ISSN: 2472-369X | www.austinpublishinggroup.com Jalal et al. (C) All rights are reserved
Citation: Jalal Y, Zaimi S, Ouzaa MR, Zine A and Jaafar A. Medial Subtalar Dislocation in a Basketball Player: Case Report and Literature Review. Austin J Orthopade \& Rheumatol. 2017; 4(3): 1060. 


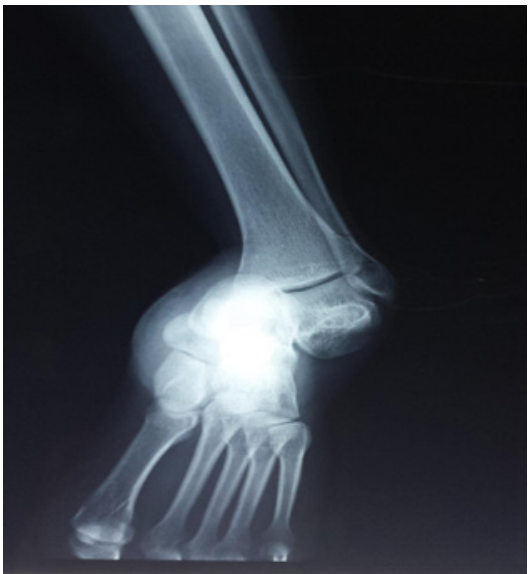

Figure 2: Anteroposterior view of a medial acute traumatic subtalar dislocation of the right ankle.

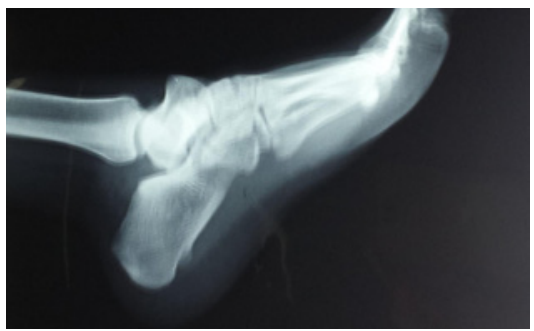

Figure 3: Lateral view of a medial acute traumatic subtalar dislocation of the right ankle.

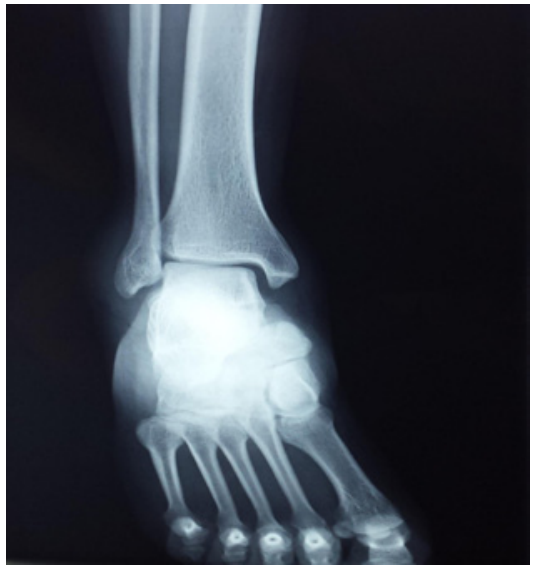

Figure 4: Anteroposterior image after closed reduction of a medial subtalar dislocation.

in 1811 were the first to describe subtalar joint dislocations [6]. Since then, the lesion has been extensively reported by several authors, and in 1852 Broca classified this injury into three types (medial, lateral and posterior). Malgaigne added the fourth type in 1856. According to a recent literature review of 359 subtalar dislocations, by Hoexum et al, $50 \%$ to $80 \%$ were caused by a high energy and just few cases result from rather trivial injuries or during sports ("basketball foot") [7]. Medial dislocation, also known as "acquired clubfoot", is the result of forceful inversion of the forefoot, which applies stress on the lateral collateral ligament when the foot is in plantarflexion. In

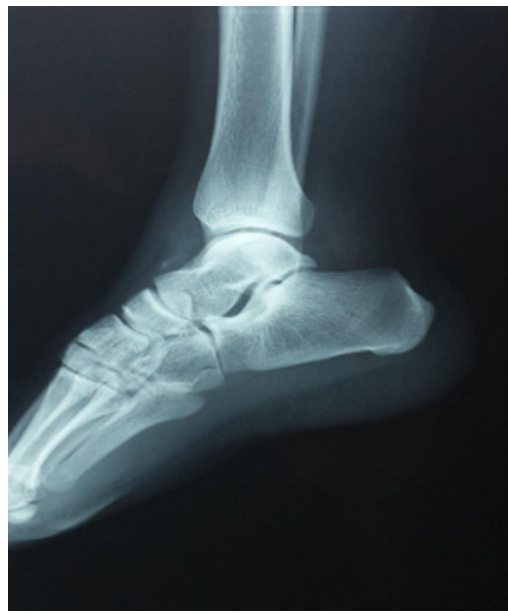

Figure 5: Lateral image after closed reduction of a medial subtalar dislocation.

contrast, lateral subtalar dislocations are produced by forced eversion with the foot in dorsiflexion [7]. However, excessive dorsiflexion of the foot usually results in an anterior dislocation of the subtalar joint, whereas excessive plantar flexion leads to posterior dislocation. In this patient, we believe the injury resulted from landing on a plantarflexed foot. Prompt closed reduction under anesthesia is the first line of management of such injury. It minimizes the soft tissue damage and reduces the incidence of a vascular necrosis of the talus. The majority of subtalar dislocations can be reduced in a closed manner under sedation. However, in $10 \%$ of medial subtalar dislocations and in $15-20 \%$ of lateral dislocations, closed reduction cannot be achieved [8]. This unsuccessful reduction in the remainder of cases was attributed to Soft tissue interposition like tibialis posterior tendon in lateral subtalar dislocation, or bony blocks like an impaction fracture of the talar head in medial subtalar dislocation. In those cases, open reduction is indicated. After closed or open reduction, numerous authors recommend CT scan to not miss relevant injuries on plain radiographs [5].

After successful reduction, the foot is immobilized in a cast. The optimal period of post reduction immobilization is still controversial. When DeLee and Curtis [9] consider that immobilization for $>3$ weeks resulted in poor outcomes, the Palma et al. [10] suggests immobilizing patients for 5 weeks in cases of isolated dislocation. For our patient, the plaster was kept for 4 weeks and the rehabilitation progresses slowly into strengthening, gait and balancing activities. Two years postoperatively, he was ambulant, and he could also return to playing sport.

Prognosis after subtalar dislocation depends on the type of injury. Although isolated subtalar dislocations with early reduction lead to excellent outcomes, less favorable results are seen with associated osseous and cartilaginous injuries. Furthermore, medial subtalar dislocations are associated with better outcomes than lateral subtalar dislocations. Another factors resulting in unsatisfactory clinical outcomes was the Open dislocations, which have worse results, unlike closed injuries.

In conclusion, this case highlights the importance of a proper diagnosis and timely management of dislocations around the subtalar joint. 


\section{Conclusion}

Isolated subtalar dislocations have an excellent prognosis with early reduction. If closed reduction is impossible because of locked dislocations or soft tissue interposition, open reduction is indicated. Because of the high incidence of associated articular fracture and associate poor prognosis, CT scan of the foot and ankle should be obtained after reduction and splinting.

\section{References}

1. Perugia D, Basile A, Massoni C, Gumina S, Rossi F, Ferretti A. Conservative treatment of subtalar dislocations. IntOrthop. 2002; 26: 56-60

2. Inokuchi S, Hashimoto T, Usami N, Ogawa K. Subtalar dislocation of the foot. Foot. 1996; 6: 168-174.

3. Malgaigne JF, Burger DC. Fractures and Sprains, Rieger, Stuttgart. 1856.

4. Jerome JT, Varghese M, SankaranB. Anteromedialsubtalar dislocation. J Foot Ankle Surg. 2007; 46: 52-54.
5. Bibbo C, Lin SS, Abidi N, Berberian W, Grossman M, Gebauer G, et al Missed and associated injuries after subtalar dislocation: the role of CT. Foot Ankle Int. 2001; 22: 324-328.

6. Shands AR. The incidence of subastragoloid dislocation of the foot with a report of one case of the inward type. J Bone Joint Surg. 1928; 10: 306-312.

7. Hoexum F, Heetveld MJ. Subtalardislocation: two cases requiring surgery and a literature review of the last 25 years. Arch Orthop Trauma Surg. 2014; 134: $1237-1249$.

8. Heppenstall RB, Farahvar H, Balderston R, Lotke P. Evaluation and management of subtalar dislocations. J Trauma. 1980; 20: 494-497.

9. DeLee JC, Curtis R. Subtalar dislocation of the foot. J Bone Joint Surg Am. 1982; 64: 433-437.

10. De Palma L, Santucci A, Marinelli M, Borgogno E, Catalani A. Clinica outcome of closed isolated subtalar dislocations. Arch Orthop Trauma Surg. 2008; 128: 593-598.
Austin J Orthopade \& Rheumatol - Volume 4 Issue 3 - 2017 ISSN: 2472-369X | www.austinpublishinggroup.com Jalal et al. () All rights are reserved
Citation: Jalal Y, Zaimi S, Ouzaa MR, Zine A and Jaafar A. Medial Subtalar Dislocation in a Basketball Player: Case Report and Literature Review. Austin J Orthopade \& Rheumatol. 2017; 4(3): 1060. 